\section{Sober-living houses and changes in the personal networks of individuals in recovery}

\author{
David G. Mueller, ${ }^{1}$ Leonard A. Jason ${ }^{2}$ \\ 1Webster University, Webster Groves, \\ MO; 2DePaul University, Chicago, IL, USA
}

\section{Abstract}

Social networks are an important source of support for many people in recovery from alcohol abuse. The present study investigated the role of one particular source of support for recovery in changing the personal networks of people in recovery, sober-living houses. In a randomized, longitudinal design changes in the network size, heterogeneity, and composition of usual aftercare and sober-living home residents were examined. Beneficial changes were found, such as increases in the number of recovering alcoholics and overall network size among sober-living home residents, particularly those who stayed six months or longer. Networks also became more homogeneous with respect to non-drinking among residents. The importance of changes in networks is discussed as well as the need for network-level analyses of personal recovery networks.

\section{Introduction}

Social support is one particular aspect of personal networks that is important for substance abuse treatment and in maintaining abstinence following treatment. Support for drinking is negatively related to abstinence for both outpatient and aftercare patients at 12 month and 3 year follow ups.1,2 Both general and alcohol-specific perceived support is negatively related to drinking,, 4 although functional alcohol-specific support is a better predictor of abstinence. ${ }^{5-7}$ Investment in social relationships is found an important moderator of the relationship between abstinence and alcoholspecific social support.7,8

Given the important connection between social support and recovery, there is a need to better understand where people can obtain these resources. Two examples of such sources are self-help/mutual aid groups and sober-living homes (SLHs). While there are a variety of SLHs, Oxford House is perhaps the most widespread with over 1,500 houses located in the United States, Australia, and Africa. 0xford House, founded in 1975 in Silver Springs, Maryland, is a democratically-run, self-sup- porting, drug free group home located in lowcrime, middle-class neighborhoods. It is unique in several ways, including that it does not staff recovery professionals and is composed of a rotating, democratically-elected leadership structure made up of house residents, residents can stay indefinitely as long as they refrain from drug or alcohol use, and residents pay about $\$ 100$ a week for rent and utilities. The $\mathrm{OH}$ network provides affordable and safe housing for recovering substance abusers and is the only substance abuse recovery home model that has been endorsed by SAMSHA as being effective. ${ }^{9}$

A NIAAA grant-supported study successfully recruited 150 individuals who completed treatment at alcohol and drug abuse facilities in the Chicago metropolitan area. Half of the participants were randomly assigned to live in $\mathrm{OHs}$, while the other half received community-based aftercare services (Usual Care). We were able to track over $89 \%$ of the $\mathrm{OH}$ and $86 \%$ of the Usual Care participants throughout the study. Results from this randomized study were encouraging and indicated significantly more successful outcomes including reduced relapse for $\mathrm{OH}$ than Usual Care participants 24 months after discharge from residential treatment. ${ }^{10}$ Positive outcomes were evident in terms of substance use (31.3\% of participants assigned to the $\mathrm{OH}$ condition reported substance use at 24 months compared to $64.8 \%$ of Usual Care participants), employment (76.1\% of $\mathrm{OH}$ participants versus $48.6 \%$ of Usual Care participants reported being employed at the 24 month assessment) and days engaged in illegal activities during the 30 days prior to the final assessment (mean $=0.9$ for $\mathrm{OH}$ and 1.8 for Usual Care participants).

Several studies investigated mechanisms of social support in Oxford House. For instance, Davis and Jason 11 found that longer lengths of stay were negatively associated with support for alcohol or drug use, while Jason et al.12 found greater sobriety among Oxford House residents with lower functional support for alcohol or drug use. Others extended this research to various subgroups. Flynn et al.13 found among African American residents that many abstinent personal networks consisted of Oxford House residents. Both Kim et al. ${ }^{14}$ and Ortiz et al. 15 found benefits for men of having children in their support networks, including number of recovery attempts, decreased substance use, and increased general social support. These types of support networks might help us better understand recovery house effectiveness, such as resultant bonding, monitoring, goal direction, modeling, positive reinforcement, rewarding alternatives to using, and advice and outlets for dealing with negative emotions and stress. Since relationships within the house (and/or in the personal network outside the house) are likely to be vehi-
Correspondence: David G. Mueller, 470 E Lockwood Ave, Webster Groves, M0 63119, USA. Tel. +1.314.968.6900.

Email: muelledg@yahoo.com

Key words: social networks, sober living house, social model.

Acknowledgements: the authors express gratitude to Stephanie Marez and Kristin Mauldin.

Contributions: DM and LAJ designed the study, analyzed and interpreted the data, and wrote the paper.

Conflict of interests: the authors declare no potential conflict of interests.

Funding: the authors would like to acknowledge the funding provided for this project from the National Institute on Alcohol Abuse and Alcoholism (R01AA12218) and the National Institute of Drug Abuse (R01DA19935).

Received for publication: 28 January 2013.

Revision received: 22 February 2013.

Accepted for publication: 22 February 2013.

This work is licensed under a Creative Commons Attribution NonCommercial 3.0 License (CC BYNC 3.0).

(OC) Copyright D.G. Mueller and L.A. Jason, 2014 Licensee PAGEPress, Italy

Health Psychology Research 2014; 2:988

doi:10.4081/hpr.2014.988

cles for these processes, integration can be viewed as relationship formation processes.

Research on social networks and support in SLHs has not investigated the role of network size and network heterogeneity. Composite functional indexes fail to capture the unique role these variables may have.12,16 Similarly, research on Oxford House has also not investigated the unique role of network composition variables, such as the presence (or absence) of relationships with drinkers and non-drinkers.

The size of one's personal network reflects the influence of the individual, their social engagement, and access to resources and materials goods. Larger networks are associated with increased availability of support resources, 17 better health, and reduction in stress response. ${ }^{18}$ Previous research has shown that Oxford House is associated with increased support resources, ${ }^{11}$ but it has not been linked to changes in structural aspects of personal networks.

Structural network heterogeneity is the mixture of drinkers and non-drinkers in a network. Sober living houses may affect this mixture. Social network analysis has developed several metrics to represent network-level characteristics. ${ }^{19}$ One kind of network-level 
metric, structural network heterogeneity, has not been examined in social support research on recovery from substance abuse. Residence in a sober-living house means living with others who are in recovery, but drinkers may still remain in a personal network for a variety of reasons, including motivation and family-ties. Moreover, living with others who are in recovery may not necessarily result in assimilation into the network. The number of relationships with certain kinds of individuals (e.g. drinkers) may also be important. For example, the number of people in the network that are heavy drinkers. Network-level metrics, such as heterogeneity, are focused on understanding the emergent properties of embedded social behavior. An approach closer to usual social support research is to examine the presence or absence of relationships held by the respondent. Such individual-level measures of network composition are functional indicators of support. Persistent or new relationships with drinkers may indicate lack of support for sobriety, while new relationships with non-drinkers may provide support mechanisms that aid recovery. We expected Oxford House to be associated with beneficial changes in personal networks. Specifically, we hypothesized that the size of personal networks would increase networks would become less heterogeneous with respect to drinkers heavy, moderate, and light drinking network members would be released; and abstinent and recovering alcoholic network members would be retained or acquired among 0xford House residents over time when compared with aftercare as usual. The second hypothesis involves length of stay. It was hypothesized that the size of personal networks would increase; networks would become less heterogeneous with respect to drinkers; heavy, moderate, and light drinking network members would be released; and abstinent and recovering alcoholic network members would be retained or acquired among Oxford House residents with lengths of stay of six months or longer when compared to lengths of stay less than six months.

\section{Materials and Methods}

\section{Participants}

Participants were recruited over a one-anda-half year period to allow a gradual transition of individuals into both conditions. Data were collected from 2002 to 2005 . In order to participate in the study, in-patient clients needed to agree to be assigned randomly to an 0xford House or usual after-care condition. Of those people approached to be in the study, only four individuals indicated that they were not interested in being involved in the project. A total of 150 adults approached at treatment centers agreed to participate, signed consent forms, and were assigned randomly to either one of the two conditions. There were 75 adults (46 women, 29 men) in the 0xford House condition and 75 (47 women, 28 men) in the usual aftercare condition.

Alcohol dependence is considered a chronic mental health disorder with relapses, and by studying substance use over this period of time, we had a better gauge of whether the person was able to cease abusing alcohol and drugs over a period of time. For each 6 month assessment, we were able to assess whether or not the person was using, and this was one of our main outcome measures of recovery. Participants assigned to usual aftercare chose a living situation that they, in conjunction with a case manager, would have made without the study. In most instances, participants lived with friends or family, moved to a non-0xford House recovery home, or moved to a single occupancy residence.

\section{Procedure}

All participants took part in a baseline interview 2-3 days prior to discharge from their treatment programs. After the baseline measure, participants were interviewed every 6 months for a 2-year period, yielding a total of five assessments (i.e., baseline, $6,12,18$, and 24 month follow-ups). To reach the participants across the assessment waves, interviewers used data from a detailed tracking packet developed for this study. Participants were paid $\$ 40$ for filling out the baseline battery, and the same amount was used for all subsequent waves. The completion rate across the 2 -year period was comparable for participants in the Oxford House (89\%) and usual aftercare (86\%) conditions. After completion of the baseline battery, participants were randomly assigned to one of the two conditions. Participants assigned to Oxford House were brought by research staff to one of the 20 existing Illinois houses. Their entrance as a resident in the house depended on a majority vote by all existing house residents. All Oxford House participants except one were successfully voted into a house on the first attempt. The single participant who was not voted into the first house was brought to a second, and was then successfully accepted as a resident. Over the course of the study, two individuals assigned to the usual aftercare condition applied for and gained admission to an 0xford House (both decided to apply for entry after spending time at other sites following discharge from the treatment facility). Using intent-to-treat rules, both individuals continued to be assigned to the usual care condition until the end of the study.

\section{Measures}

Substance use data was collected through the Addiction Severity Index 20 and personal network data was collected with the Important People Inventory. ${ }^{21}$ Demographic data was collected with an ad-hoc instrument.

The Addiction Severity Index-lite (ASI) ${ }^{20}$ is a reliable and well validated instrument that assesses problem areas commonly related to substance abuse including medical status, drug use, alcohol use, illegal activity, family relations, family history, and psychiatric condition. The instrument was administered at every assessment. Prior 30 day alcohol use was obtained from this instrument. The alcohol use outcome variable was coded dichotomously ( 0 $=$ no alcohol consumed in the last 30 days, 1 = one or more drinks in the last 30 days).

The Important People Inventory $(I P)^{21,22}$ was administered at each wave. This structured interview is a modified version of the Important People and Activities Inventory, such that the items that comprise the Activities portion are omitted. Because this assessment tool has various adaptations and administration manuals, it is important to note that the version administered in this study was adapted from the edition developed by Clifford and Longabaugh for use in Project MATCH. The IP requires participants to identify important members in their networks with whom they have had frequent contact with in the past 6 months. In the first section of the IP, labeled the Important People section, a participant is asked to identify up to 12 social contacts over the age of 12 years. For each person the participant lists in his/her network the measure examines the type of relationship (e.g., spouse, parent, friend, co-worker), the duration of relationship in years, and the frequency of contact. In addition, the participant assesses how often the network member drinks, how much the network member drinks on a maximum drinking day, and the network member's overall drinking status (i.e., heavy, moderate, light, abstainer, or recovering). In the next section, called the Most Important People (MIP) section, the participant chooses up to four network members who were the most important over the past six months. The participant then rates each network member's importance, how much he/she likes the person, and how the person reacts to the participant's drinking. The MIP portion of the instrument was omitted from analysis in the present study.

There are seven dependent variables. The first is personal network size, which was obtained by summing the number of alters provided and adding one (for the respondent).

The second dependent variable, network heterogeneity, was obtained using Blau's index of heterogeneity: ${ }^{23}$

$$
H=\left(1-\Sigma^{2}{ }_{i}\right)
$$

where $\mathrm{p}$ is the proportion of network members in a category and $i$ is the number of different 
categories represented in a network. The categories in this analysis were the number of heavy drinkers, moderate drinkers, light drinkers, abstainers, and recovering alcoholics. High levels of network heterogeneity $(H$ values closer to 1 ) indicate a mixture of drinkers and non-drinkers, while low levels indicate homogeneity with respect to drinkers or non-drinkers ( $H$ values closer to 0 ).

The third through seventh dependent variables were composition variables at each wave. The third variable was the total number of heavy drinkers, the fourth was the total number of moderate drinkers, the fifth was the total number of light drinkers, the sixth was the total number of alcohol abstainers, and the seventh was the total number of recovering alcoholics.

Inspection of the empirical growth plots for several of the dependent variables revealed second degree polynomial curves. Appropriate quadratic representations of time were entered in the model where applicable. All other models used a linear representation of the wave trajectory.

\section{Results}

Across both conditions, most participants were women (62\%). The sample consisted of 77.3\% African American participants, 11.3\% European American, 8\% who were Hispanic/Latino American, and 3.3\% Asian American. The average age of participants at baseline was 37.03 years (SD = 7.95). The average education at baseline was 11.95 years $(\mathrm{SD}=2.05)$. The sample had a life-time average of $3.2(\mathrm{SD}=2.1)$ episodes of in-patient and $0.8(\mathrm{SD}=2.6)$ episodes of out-patient substance abuse treatment. Over the 2-year follow-up, Oxford House participants spent an average of 256.2 days (range 8-730) in this setting. Of the 75 Oxford House participants, 5\% stayed in Oxford House for the entire 24 months of the study, $35 \%$ moved into their own home or apartment after leaving the Oxford House, 20\% went to relatives' homes, $15 \%$ moved into a partner's or spouse's home, $9 \%$ went to a friend's home, $5 \%$ went to a treatment program, $4 \%$ went to jail, $4 \%$ went to another staffed recovery home, and $3 \%$ went to a homeless shelter. Baseline differences between participants in the two conditions were first evaluated by chi-square, independent sample t-tests, or zero-order correlations, depending on the nature of the variable and the scale used. Results indicated no significant differences between participants in either Oxford House or usual-care conditions on demographic variables.

Characteristics of personal networks at baseline were also computed. Networks were composed of $66 \%$ females and $33 \%$ males. Relationship type was also computed with family members making up $70 \%$ of networks, intimates/ex-intimates accounted for 9\%, and associates (i.e. friends and co-workers) consisted of $20 \%$ of networks. Participants reported knowing their network for an average of 24.18 years $(\mathrm{SD}=9.39)$. Participants also reported that alters were very supportive with a network average of $5.40(\mathrm{SD}=0.68)$ on a scale from 1 (not at all supportive) to 6 (extremely supportive). Participants reported moderate frequency of contact with alters with a network average of $4.87(\mathrm{SD}=1.78)$ on a scale from 0 (no contact) to 7 (daily contact).

There is an expectation with the utilization of random assignment that participants will not differ by treatment condition at baseline. Results confirmed this expectation for all dependent variables.

\section{Treatment condition changes in composition, heterogeneity, and size}

Hierarchical linear modeling was used in all analyses. The linear and/or quadratic wave trajectory defined by each 6 -month time period was included as a Level-l variable. Age was also included as a time-varying Level-1 variable. Treatment condition (Oxford House versus usual aftercare), gender, and education at baseline were entered as Level-2 variables. Network composition, heterogeneity, and size were outcome variables.

In the first model, the gammas for the second level variable, treatment condition, predicting the relationship between time and the network composition dependent variables were significant for the number of recovering alcoholics in the network and the number of heavy drinkers in the network.

We found a significant amount of variability around the dependent variables number of recovering alcoholics $\left(\sigma^{2}=0.84, \chi^{2}(145)=\right.$ 575.68, $\mathrm{P}<0.001)$ and number of heavy drinkers $\left(\sigma^{2}=0.84, \chi^{2}(146)=580.10\right.$, $\mathrm{P}<0.001)$. Hence, a random intercept was estimated in each model.

Results indicated steeper increases were found for Oxford House compared to usual aftercare participants in the number of people in the network who were recovering alcoholics $(b=0.14, \quad \mathrm{SE}=0.06, \quad t(660)=2.34, \quad \mathrm{P}=0.02)$. Analysis also indicated that the number of heavy drinkers $(b=-0.05, \mathrm{SE}=0.02, t(660)=-$ 2.91, $\mathrm{P}=0.004$ ) for usual aftercare and 0xford House participants was statistically significant (Figure 1). Oxford House residents did not change in the number of heavy drinkers in the network over time, while usual aftercare participants saw increases in the number of heavy drinkers. The remaining variables were not significantly predicted by treatment condition: the number of alcohol abstainers $(b=-0.26$,
$\mathrm{SE}=0.32, t(656)=-0.79, \mathrm{P}=0.42)$, the number of light drinkers $(b=-0.01, \mathrm{SE}=0.03, t(656)=$ $0.63, \mathrm{P}=0.52)$, the number of moderate drinkers $(b=0.01, \quad \mathrm{SE}=0.01, \quad t(656)=0.72$, $\mathrm{P}=0.46)$, network heterogeneity $(b=0.01$, $\mathrm{SE}=0.01, t(656)=0.21, \mathrm{P}=0.83)$, and network size $(b=0.11, \mathrm{SE}=0.40, t(656)=0.29, \mathrm{P}=0.76)$.

\section{Length-of-stay changes in compo- sition, heterogeneity, and size}

For the second model, the linear and/or quadratic wave trajectory defined by each 6 month time period was included as a Level-l variable. Age was also included as a time-varying Level-1 variable. Length-of-stay (six months or greater or less than six months), gender, and education at baseline were entered as Level-2 variables. Network composition, heterogeneity, and size were outcome variables.

For this model we were interested in how length of stay in Oxford House affects changes in composition, network size, and heterogeneity. Among Oxford House residents, 54.8\% of the sample had lived in a house for less than 6 months, while the remaining $45.2 \%$ had lived in a house for more than 6 months.

We found a significant amount of variability around the dependent variables number of recovering alcoholics $\left(\sigma^{2}=0.71\right.$, $2(71)=213.32, \mathrm{P}<0.001)$, number of light drinkers $\left(\sigma^{2}=0.47,2(71)=350.69, \mathrm{P}<0.001\right)$, network heterogeneity $\left(\sigma^{2}=0.01\right.$, $2(71)=153.32, \mathrm{P}<0.001)$, and network size $\left(\sigma^{2}\right.$ $\left.=1.11, \chi^{2}(71)=239.91, \mathrm{P}<0.001\right)$. Hence, $\mathrm{a}$ random intercept was estimated in each model.

The gammas for the second level length-ofstay variable predicting the relationship between time and number of recovering alcoholics $\quad(b=0.36, \quad \mathrm{SE}=0.09, \quad t(330)=3.74$, $\mathrm{P}=0.001)$ and time and number of light drinkers $(b=-0.15, \mathrm{SE}=0.05, t(330)=-2.74$, $\mathrm{P}=0.007$ ) were statistically significant (Figure 2 ). A length of stay of six months or longer was associated with steeper increases in the number of recovering alcoholics over time when compared with a length of stay less than six months. On the other hand, a length of stay of six months or longer was associated with decreases in the number of light drinkers, while the number of light drinkers did not change for lengths of stay less than six months.

Interestingly, the gamma for the length-ofstay variable predicting the relationship between age and number of heavy alcoholics was also statistically significant $(b=0.01$, $\mathrm{SE}=0.005, t(71)=2.63, \mathrm{P}=0.01)$. When length of stay is greater than six months there is an increase in the number of heavy alcoholics in the personal network as age increases, but when length of stay is less than six months there is a decrease in the number of heavy 
alcoholics as age increases.

There were main effects of linear $(b=-0.32$, $\mathrm{SE}=0.14, t(326)=-2.23, \mathrm{P}=0.02)$ and quadratic $(b=0.05, \mathrm{SE}=0.02, t(326)=2.53, \mathrm{P}=0.01)$ time on network heterogeneity. Network heterogeneity decreased between waves 1 and 2 of the study, and then increased at each subsequent wave for all 0xford House residents. The gamma for the second level length of stay variable predicting the relationship between linear time and network heterogeneity $(b=0.16$, $\mathrm{SE}=0.08, t(326)=2.03, \mathrm{P}=0.04)$ and quadratic time and network heterogeneity $(b=-0.02$, $\mathrm{SE}=0.01, t(326)=-2.27, \mathrm{P}=0.02)$ was also significant (Figure 2). Network heterogeneity initially increased for residents with lengths of stay of six months or greater and decreased for residents with lengths of stay less than six months. However, this pattern reversed at every subsequent wave where residents with lengths of stay of six months or greater experienced a decrease in network heterogeneity while residents with a length of stay less than six months experienced an increase in network heterogeneity.

Results also indicated a significant main effect of both linear $(b=-2.51, \mathrm{SE}=0.95$, $t(326)=-2.64, \mathrm{P}=0.01)$ and quadratic $(b=0.39$, $\mathrm{SE}=0.41, t(326)=2.67, \mathrm{P}=0.01)$ change in network size. The linear effect of time indicates that network size decreases at the first wave, but that it increases at each subsequent wave for all Oxford House residents. The gamma for the length of stay variable significantly predicted the relationship between linear time and network size $(b=1.29, \mathrm{SE}=0.59, t(326)=2.16$, $\mathrm{P}=0.03$ ) and quadratic time and network size $(b=-0.19, \mathrm{SE}=0.09, t(326)=-2.04, \mathrm{P}=0.04)$. For participants who spent six months or more in 0xford House there was an initial mild increase in personal network size at wave 1 followed by a mild decrease in personal network size at each subsequent wave. Participants who spent less than six months in Oxford House experienced an initial strong decrease in the size of their personal network at wave 1 and strong increases in network size at each subsequent wave.

The remaining variables were not significantly predicted by length of stay: number of abstainers $(b=-0.02, \mathrm{SE}=0.09 \quad t(330)=-0.20$, $\mathrm{P}=0.84)$, number of moderate drinkers $(b=0.01, \mathrm{SE}=0.04 t(330)=0.46, \mathrm{P}=0.64)$, and number of heavy drinkers $(b=-0.01, \mathrm{SE}=0.02$ $t(330)=-0.70, \mathrm{P}=0.48)$.

\section{Discussion}

Marked changes in the structure of the personal networks of residents in sober-living homes is evident. Overall, Oxford House seems to be related to changes in the composition of personal networks of people in recovery from alcohol abuse. Specifically, it is associated with increases in the number of recovering alcoholics and seems to protect against the addition of heavy drinking network members. Length of stay in Oxford House was also associated with significant changes to structural characteristics of personal networks. The overall size of the personal network and the number of recovering alcoholics increased, while the number of light drinkers decreased for residents who stayed six months or longer. Networks also became more homogeneous generally moving from a mixture of drinkers and non-drinkers to mostly non-drinkers among residents who stayed six months or longer. This study also presented findings indicating that several variables changed quadratically suggesting that the first six months in recovery are perhaps most important for soberliving home residents. To date, research on personal networks in recovery has been limited by its attributional ego-centric approach to social support, a point that has been lamented by several researchers. 24,25 This has prevented the investigation of the important emergent properties of personal networks of which network heterogeneity is one. ${ }^{19}$ The widespread use of the Important People Inventory is one example. The data architecture of this instrument does not allow for ties between network members. It also relies on the respondent's reporting of network member behavior, such as drinking and drug use, and places an emphasis on strong ties. Our data indicate that most networks in the present study were highly supportive family members whom the respondent had known for a long time. While this is beneficial for people in recovery, it fails to fully capture the positive and/or negative influence of network members who continue to use or those who are weak ties. Utilization of the techniques of social network analysis could allow researchers to investigate a host of phenomenon that may occur in SLHs, such as the transmission of recovery and the role of social influence and social engagement in recovery.

The results of this research may be applicable to other types of SLHs. The Alcohol Research Group, for example, focused on sober living houses throughout California. These houses typically experience greater variability in their operational and social models than Oxford Houses, ${ }^{26}$ but are otherwise similar with respect to 12 -step meeting attendance

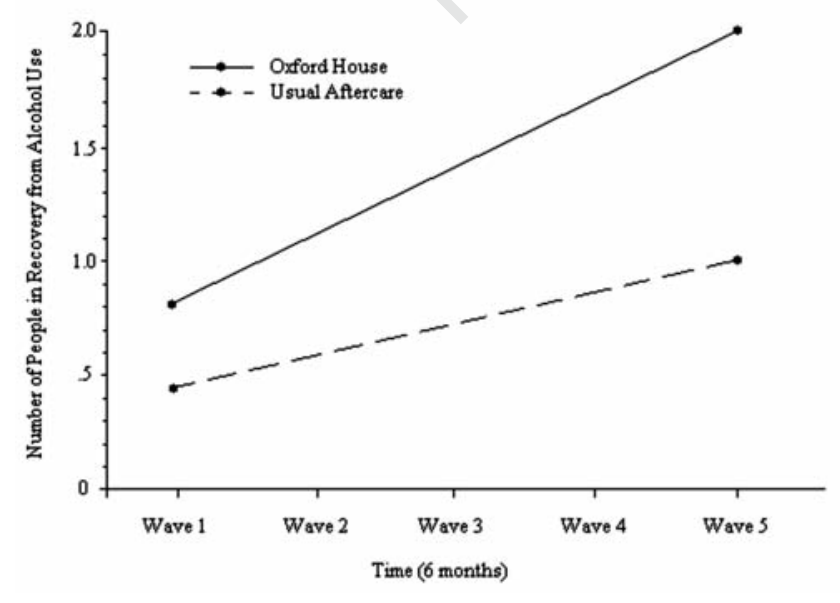

Figure 1. Change in number of recovering alcoholics for Oxford House and usual aftercare conditions.

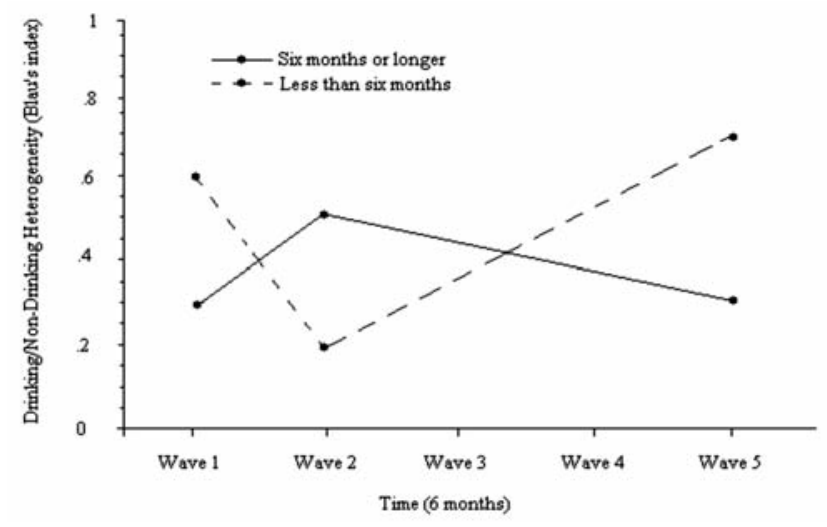

Figure 2. Change in drinking heterogeneity by length of stay. 
requirements, demographic residential makeup, and substance use history. Polcin et al.16 found increased alcohol and drug use among SLH residents with networks composed of frequently contacted individuals who used alcohol and/or drugs regularly. Polcin et al.27 found similar results for SLH residents who also attended outpatient treatment.

A natural question is whether 0xford houses are appropriate for all alcohol dependent populations, for multi-drug users, for dually diagnosed persons that show co morbidity with another severe alcohol disorder for example depression, bipolar disorder, schizophrenia, and personality disorder. Oxford Houses and other types of sober living homes are for individuals who can live independently, and if they have untreated serious mental disorders, it would be unlikely that they would be able to maintain gainful employment to reside in these houses or be able to keep up with regular tasks and responsibilities (such as chores) that are required for staying in these homes. However, our research does indicate that Oxford Houses have been successful for a variety of different populations, including those with eating disorders, ${ }^{28}$ those with gambling disorders, ${ }^{29}$ those with aggressive and criminal behaviors, 30 those with PTSD, 31 those with psychiatric disorders, ${ }^{32}$ and veterans. 33

We do not believe that social network changes alone treat alcoholism and substance abuse. Clearly, the individuals in our sample were using a wide variety of wrap-around services, including the treatment facilities from which they were recruited. Recovery homes play one part within the larger treatment ecology, and when networks do change to support recovery, they need to be supported by an infrastructure of jobs, services, and other support programs in their community. The average stay within an Oxford House is less than a year, so when leaving this recovery home, the residents often move in their own home or apartment, live with relatives or friends, move into a partner's or spouse's home, or go to a treatment program, jail, another staffed recovery home, or a shelter. There is a need for multiple programs to serve these individuals and support their efforts to be abstinent.

\section{Conclusions}

There are a number of limitations in this study. First, while we have data for a two year period of time, it certainly would have been better to assess changes for a longer period of time, such as five years, however, funding limitations prevented us from collecting more long term outcome data. Also, the majority of our participants were women and most of our participants were African-American. This certainly is not the typical profile of people in the US with substance use disorders. Because our sample was recruited from treatment facilities in Chicago, it had far more women and minorities than a representative sample, and this is another limitation in the study as it reduces the generalizability of our findings to a national population. Numerous studies have documented that social relationships influence both physical and psychological outcomes, from longevity 34 and recovery from health ailments such as cardiovascular disease 35 to the spread of infectious disease. 36 Personal social networks were widely implicated in this research for both protective and adverse effects. ${ }^{37,38}$ The present study extends this literature by showing that soberliving homes like Oxford House provide a safe, supportive, and stable living environment for people seeking recovery from alcohol abuse. The results from this study indicate that residence in Oxford House is associated with important changes in the nature of the personal networks of residents and that these changes are related to decreased relapse rates. Additional research is needed to investigate the manner in which social models of recovery like Oxford House operate.

\section{References}

1. Longabaugh R, Wirtz PW, Zweben A. Network support for drinking, alcoholics anonymous and long-term matching effects. Addiction 1998;93:1313-33.

2. Project Match Research Group. Matching alcoholism treatments to client heterogeneity: project MATCH posttreatment drinking outcomes. J Stud Alc 1997;58:729.

3. Rice C, Longabaugh R. Measuring general social support in alcoholic patients: short forms for perceived social support. Psychol Addict Behav 1996;10:104-14.

4. Gordon AJ, Zrull MC. Social networks and recovery: one year after inpatient treatment. J Subst Abuse Treat 1991;8:143-52.

5. Beattie MC, Longabaugh R. Interpersonal factors and post-treatment drinking and subjective well-being. Addiction 1997;92: 1507-21.

6. Havassy BE, Hall SM, Wasserman DA. Social support and relapse: commonalities among alcoholics, opiate users and cigarette smokers. Addict Behav 1991;16:23546.

7. Longabaugh R, Beattie M, Noel N, et al. The effect of social investment on treatment outcome. J Stud Alcohol 1993;54:46578.

8. Longabaugh R, Wirtz P, Beattie MC, et al. Matching treatment focus to patient social investment and support: 18-month follow- up results. J Consult Clin Psychol 1995;63: 296-307.

9. SAMSHA's National Registry of EvidenceBased Programs and Practices Oxford House; 2011. Available form: http://www. nrepp.samhsa.gov/ViewIntervention.aspx? id $=223$.

10. Jason LA, Olson BD, Ferrari JR, Lo Sasso AT. Communal housing settings enhance substance abuse recovery. Am J Public Health 2006;96:1727-9.

11. Davis MI, Jason LA. Sex differences in social support and self-efficacy within a recovery community. Am J Community Psychol 2005;36:259-74.

12. Jason LA, Davis M, Ferrari JR. The need for substance abuse aftercare: a longitudinal analysis of Oxford House. Addict Behav 2007;32:808-13.

13. Flynn AM, Alvarez J, Jason LA, et al. African American 0xford Houses residents: sources of abstinent social networks. J Prev Interv Community 2006; 31:111-20.

14. Kim KL, Davis MI, Jason LA, Ferrari JR. Structural social support: impact on adult substance use and recovery attempts. J Prev Interv Community 2006;31:85-94.

15. Ortiz E, Alvarez J, Jason LA, et al. Abstinence social support: the impact of children in Oxford House. J Groups Addict Recover 2009;4:71-81.

16. Polcin DL, Korcha RA, Bond J, Galloway G. Sober living house for alcohol and drug dependence: 18 month outcomes. J Subst Abuse Treat 2010;38:356-65.

17. Wellman B, Gulia M. The network basis of social support: a network is more than the sum of its ties. In: Wellman B, ed. Networks in the global village. Boulder, C0: Westview Press; 1999. pp 83-118.

18. Uchino BN, Uno D, Holt-Lunstad J, Flinders JB. Age-related differences in cardiovascular reactivity during acute psychological stress in men and women. $J$ Gerontol B Psychol Sci Soc Sci 1999;54: $339-46$.

19. Wasserman S, Faust K. Social network analysis: methods and applications. Cambridge: Cambridge University Press; 1994.

20. McLellan AT, Cacciola J, Kushner H, et al. The fifth edition of the addiction severity index: cautions, additions and normative data. J Subst Abuse 1992;9:461-80.

21. Clifford PR, Longabaugh R. Manual for the administration of the important people and activities instrument. Adapted for use by Project MATCH for NIAAA 5 R01AA06698-05 Environmental Treatment of Alcohol Abusers. Richard Longabaugh, Principal Investigator; 1991.

22. Clifford PR, Longabaugh R, Beattie M. Social support and patient drinking: A val- 
idation study. Alcohol Clin Exp Res 1992;16:403.

23. Blau PM. Inequality and heterogeneity: a primitive theory of social structure. New York: Free Press; 1977.

24. Berkman LF, Glass T. Social integration, social networks, social support, and health. In: Berkman LF, Kawachi I, eds. Social epidemiology. New York: Oxford University Press; 2000. pp 137-73.

25. Smith KP, Christakis NA. Social networks and health. Annu Rev Sociol 2008;34:40529.

26. Polcin DL, Henderson D. A clean and sober place to live: philosophy, structure, and purported therapeutic factors in sober living houses. J Psychoactive Drugs 2008;40:153-9.

27. Polcin DL, Korcha R, Bond J, Galloway G. Eighteen month outcomes for clients receiving outpatient treatment and Sober Living Houses. J Subst Use 2010;15:35266.

28. Czarlinski JA, Aase DM, Jason LA. Eating disorders, normative eating self-efficacy and body image self-efficacy: women in recovery homes. Eur Eat Disord Rev 2012;20:190-5.

29. Majer JM, Angulo RS, Aase DM, Jason LA. Gambling behaviors among Oxford House residents: a preliminary investigation. $\mathrm{J}$ Soc Serv Res 2011;37:422-7.

30. Aase DM, Jason LA, Olson BD, et al. A longitudinal analysis of criminal and aggressive behaviors among a national sample of adults in mutual-help recovery homes. In: Jason LA, Ferrari JR, eds. Recovery from addiction in communal living settings: the Oxford House model [Special Issue]. J Groups Addict Recovery 2009;4:82-91.

31. Jason LA, Mileviciute I, Aase DM, et al. How type of treatment and presence of PTSD affect employment, self-regulation, and abstinence. N Am J Psychol 2011;13: 175-186.

32. Majer JM, Jason LA, North CS, et al. A longitudinal analysis of psychiatric severity upon outcomes among substance abusers residing in self-help settings. Am $\mathrm{J}$ Community Psychol 2008;42:145-53.
33. Millar J, Aase DM, Jason LA, Ferrari JR. Veterans residing in self-governed recovery homes for substance abuse: sociodemographic and psychiatric characteristics. Psychiatr Rehabil J 2011;35:141-4.

34. Berkman LF, Syme SL. Social networks, host resistance and mortality: a nine year follow-up study of Alameda County residents. Am J Epidemiol 1979;109:186-204.

35. Orth-Gomer K, Rosengren A, Wilhelmsen L. Lack of social support and incidence of coronary heart disease in middle-aged Swedish men. Psychosom Med 1993;55:3743.

36. Morris M. Data driven network models for the spread of infectious disease. In: Mollison D, ed. Epidemic models: their structure and relation to data. Cambridge, UK: Cambridge University Press, 1995.

37. Berkman LF. The role of social relations in health promotion. Psychosom Med 1995; 57:245-54

38. Seeman TE. Social ties and health: the benefits of social integration. Ann Epidemiol 1996;6:442-51. 